\title{
Riccati Equations and Delay-Dependent BIBO Stabilization of Stochastic Systems with Mixed Delays and Nonlinear Perturbations
}

\author{
Xia Zhou and Shouming Zhong \\ School of Mathematical Sciences, University of Electronic Science and Technology of China, \\ Chengdu, Sichuan 611731, China \\ Correspondence should be addressed to Xia Zhou, zhouxia44185@sohu.com
}

Received 21 August 2010; Accepted 9 December 2010

Academic Editor: T. Bhaskar

Copyright (C) 2010 X. Zhou and S. Zhong. This is an open access article distributed under the Creative Commons Attribution License, which permits unrestricted use, distribution, and reproduction in any medium, provided the original work is properly cited.

\begin{abstract}
The mean square BIBO stability is investigated for stochastic control systems with mixed delays and nonlinear perturbations. The system with mixed delays is transformed, then a class of suitable Lyapunov functionals is selected, and some novel delay-dependent BIBO stabilization in mean square criteria for stochastic control systems with mixed delays and nonlinear perturbations are obtained by applying the technique of analyzing controller and the method of existing a positive definite solution to an auxiliary algebraic Riccati matrix equation. A numerical example is given to illustrate the validity of the main results.
\end{abstract}

\section{Introduction}

In recent years, Bounded-Input Bounded-Output (BIBO) stabilization has been investigated by many researchers in order to track out the reference input signal in real world, see [1-6] and some references therein. On the other hand, because of the finite switching speed, memory effects, and so on, time delays are unavoidable in technology and nature, commonly exist in various mechanical, chemical engineering, physical, biological, and economic systems. They can make the concerned control system be of poor performance and instable, which cause the hardware implementation of the control system to become difficult. It is necessary to introduce the distributed delay in control systems, which can describe mathematical modeling of many biological phenomena, for instance, in preypredator systems, see [7-9]. And so, BIBO stabilization analysis for mixed delays and nonlinear systems is of great significance.

In $[10,11]$, the sufficient condition for BIBO stabilization of the control system with no delays was proposed by the Bihari-type inequality. In $[12,13]$, employing the parameters 
technique and the Gronwall inequality investigated the BIBO stability of the system without distributed time delays. In [14-16], based on Riccati-equations, by constructing appropriate Lyapunov functions, some BIBO stabilization criteria for a class of delayed control systems with nonlinear perturbations were established. In [17], the BIBO stabilization problem of a class of piecewise switched linear system was further investigated. However, up to now, these previous results have been assumed to be in deterministic systems, including continuous time deterministic systems and discrete time deterministic systems, but seldom in stochastic systems (see [18], Fu and Liao get several mean square BIBO stabilization criteria in terms of Razumikhin technique and comparison principle). In practice, stochastic control systems are more applicable to problems that are environmentally noisly in nature or related to biological realities. Thus, the BIBO stabilization analysis problems for stochastic case are necessary.

Up to now, to the best of authors knowledge, the method of existence of a positive definite solution to an auxiliary algebraic Riccati matrix equation is only used to deal with the BIBO stabilization for deterministic differential equations [14-17], not for stochastic differential equations.

Motivated by the above discussions, the main aim of this paper is to study the BIBO stabilization in mean square for the stochastic control system with mixed delays and nonlinear perturbations. Based on the technique of analyzing controller and transforming of the system, various suitable Lyapunov functionals are selected, different Riccati matrix equations are established, and some sufficient conditions guaranteeing BIBO stabilization in mean square are obtained. Finally, a numerical example is provided to demonstrate the effectiveness of the derived results.

\section{Problem Formulation and Preliminaries}

Consider the stochastic control system described by the following equation:

$$
\begin{aligned}
d x(t)= & {\left[A x(t)+B_{1} x\left(t-h_{1}\right)+B_{2} \int_{t-h_{2}}^{t} x(s) d s+f(t, x(t))+D u(t)\right] d t } \\
& +\left[C_{1} x\left(t-\tau_{1}\right)+C_{2} \int_{t-\tau_{2}}^{t} x(s) d s\right] d \mathbf{w}(t), \quad t \geq t_{0} \geq 0, \\
y(t)= & H x(t), \\
x(\theta)= & \varphi(\theta) \in C_{q_{0}}^{b}\left(\left[t_{0}-\tau, t_{0}\right] ; R^{n}\right), \quad \theta \in\left[t_{0}-\tau, t_{0}\right],
\end{aligned}
$$

where $x(t), u(t), y(t)$ are the state vector, control input, control output of the system, respectively. $\tau_{1}>0, h_{1}>0$ are discrete time delays, and $h_{2}>0, \tau_{2}>0$ are distributed time delays, $\tau=\max \left\{\tau_{1}, \tau_{2}, h_{1}, h_{2}\right\} . A, B_{1}, B_{2}, C_{1}, C_{2}, D, H$ are constant matrices with appropriate dimensional, and $C_{2}, D$ are nonsingular matrices. $\mathbf{w}(t)=\left(\mathbf{w}_{1}(t), \mathbf{w}_{2}(t), \ldots, \mathbf{w}_{n}(t)\right)$ is an $n$-dimensional standard Brownian motion defined on a complete probability space $\left(\Omega, F,\left\{F_{t}\right\}_{t \geq 0}, P\right)$ with a natural filtration $\left\{F_{t}\right\}_{t \geq 0} . f(t, x(t))$ is the nonlinear vector-valued perturbation bounded in magnitude as

$$
\|f(t, x(t))\| \leq \alpha\|x(t)\|
$$

where $\alpha$ is known positive constant. 
To obtain the control law described by (2.1) of tracking out the reference input of the system, we let the controller be in the form of

$$
u(t)=u_{1}(t)+u_{2}(t)+u_{3}(t)
$$

with

$$
\begin{gathered}
u_{1}(t)=K_{1}(t) x(t)+r(t), \\
u_{2}(t)=K_{2}(t) x\left(t-h_{1}\right)+r\left(t-h_{1}\right), \\
u_{3}(t)=K_{3}(t) x\left(t-h_{2}\right)+r\left(t-h_{2}\right),
\end{gathered}
$$

where $K_{1}(t), K_{2}(t), K_{3}(t)$ are the feedback gain matrices, $r(t), r\left(t-h_{1}\right), r\left(t-h_{2}\right)$ are the reference inputs.

To derive our main results, we need to introduce the following definitions and lemmas.

Definition 2.1 (see [18]). A vector function $r(t)=\left(r_{1}(t), r_{2}(t), \ldots, r_{n}(t)\right)^{T}$ is said to be an element of $L_{\infty}^{n}$ if $\|r\|_{\infty}=\sup _{t \in\left[t_{0},+\infty\right)}\|r(t)\|<+\infty$, where $\|\cdot\|$ denotes the Euclid norm in $R^{n}$ or the norm of $a$ matrix.

Definition 2.2 (see [18]). The nonlinear stochastic control system (2.2) is said to be BIBO stabilized in mean square if one can construct a controller (2.5) such that the output $y(t)$ satisfies

$$
\mathbb{E}\left(\|y(t)\|^{2}\right) \leq N_{1}+N_{2}\|r\|_{\infty}^{2}
$$

where $N_{1}, N_{2}$ are positive constants.

Definition 2.3 (L-operator). Let Lyapunov functional $V: C\left([-\tau, 0] ; R^{n}\right) \times R_{+} \rightarrow R$; its infinitesimal operator, $\mathcal{L}$, acting on functional $V$ is defined by

$$
\mathcal{L} V(x(t), t)=\lim _{\Delta \rightarrow 0+} \sup \frac{1}{\Delta}[E V(x(t+\Delta), t+\Delta)-V(x(t), t)]
$$

Lemma 2.4 (see [19]). For any constant symmetric matrix $M \in R^{n \times n}, M=M^{T}>0$, scalar $r>0$, vector function $g:[0, r] \rightarrow R^{n}$, such that the integrations in the following are well defined:

$$
r \int_{0}^{r} g^{T}(s) M g(s) d s \geq\left[\int_{0}^{r} g(s) d s\right]^{T} M\left[\int_{0}^{r} g(s) d s\right] .
$$

Lemma 2.5 (see [20]). Let $x, y \in R^{n}$ and any $n \times n$ positive-definite matrix $Q>0$. Then, one has

$$
2 x^{T} y \leq x^{T} Q^{-1} x+y^{T} Q y .
$$




\section{BIBO Stabilization for Nonlinear Stochastic Systems}

Transform the original system (2.1) to the following system:

$$
\begin{aligned}
d\left[x(t)+B_{1} \int_{t-h_{1}}^{t} x(s) d s\right]= & {\left[\left(A+B_{1}\right) x(t)+B_{2} \int_{t-h_{2}}^{t} x(s) d s+f(t, x(t))\right] d t } \\
& +\left[C_{1} x\left(t-\tau_{1}\right)+C_{2} \int_{t-\tau_{2}}^{t} x(s) d s\right] d \mathbf{w}(t)+D u(t) d t, \quad t \geq t_{0} \geq 0, \\
y(t)= & H x(t), \\
x(\theta)= & \varphi(\theta) \in C_{\Psi_{0}}^{b}\left(\left[t_{0}-\tau, t_{0}\right] ; R^{n}\right), \quad \theta \in\left[t_{0}-\tau, t_{0}\right] .
\end{aligned}
$$

Theorem 3.1. The nonlinear stochastic control system (2.1) or (3.1) with the control law (2.3) is $B I B O$ stabilized in mean square if $\left\|h_{1} B_{1}\right\|<1$ and there exist symmetric positive-definite matrices $R_{i}>0, i=1,2, \ldots, 10$, and $Q_{1}>0$ such that

$$
\lambda_{\min }\left(Q_{1}\right)-2 \alpha\|P\|>0
$$

and $P$ is the symmetric positive solution of the Riccati equation

$$
P\left(A+B_{1}\right)+\left(A+B_{1}\right)^{T} P+P \Sigma_{1} P+\Xi_{1}+\Delta_{1}=-Q_{1},
$$

where

$$
\begin{aligned}
\Sigma_{1}= & B_{2} R_{1}^{-1} B_{2}^{T}+2 D R_{10} D^{T}+R_{5}^{-1}+R_{6}^{-1}+D R_{10} D^{T} P B_{1} R_{7}^{-1} B_{1}^{T} P D R_{10} D^{T} \\
& +B_{1} R_{8}^{-1} B_{1}^{T}+B_{1} R_{9}^{-1} B_{1}^{T} \\
\Xi_{1}= & h_{2}^{2}\left(R_{1}+R_{3}\right)+h_{1}^{2}\left(R_{2}+R_{7}+R_{8}+R_{9}\right)+R_{5}+R_{6}+\tau_{2}^{2} R_{4}, \\
\Delta_{1}= & \left(A+B_{1}\right)^{T} P B_{1} R_{2}^{-1} B_{1}^{T} P\left(A+B_{1}\right)+C_{1}^{T} P C_{1}+C_{1}^{T} P C_{2} R_{4}^{-1} C_{2}^{T} P C_{1} \\
& +\tau_{2}^{2} C_{2}^{T} P C_{2}+h_{1}^{2} B_{1}^{T} P B_{2} R_{3}^{-1} B_{2}^{T} P B_{1}, \\
K_{1}= & R_{10} D^{T} P, \quad K_{2}=K_{3}=D^{-1} .
\end{aligned}
$$

Proof. We define a Lyapunov functional $V(t, x(t))$ as

$$
V(t, x(t))=V_{1}(t, x(t))+V_{2}(t)+V_{3}(t)+V_{4}(t)+V_{5}(t)+V_{6}(t)+V_{7}(t),
$$


where

$$
\begin{aligned}
V_{1}(t, x(t)) & =\left(x(t)+B_{1} \int_{t-h_{1}}^{t} x(s) d s\right)^{T} P\left(x(t)+B_{1} \int_{t-h_{1}}^{t} x(s) d s\right) \\
V_{2}(t) & =\int_{t-h_{1}}^{t} x^{T}(s)\left(R_{5}+P B_{1} R_{8}^{-1} B_{1}^{T} P\right) x(s) d s \\
V_{3}(t) & =\int_{t-h_{2}}^{t} x^{T}(s)\left(R_{6}+P B_{1} R_{9}^{-1} B_{1}^{T} P\right) x(s) d s, \\
V_{4}(t) & =\int_{t-\tau_{1}}^{t} x^{T}(s)\left(C_{1}^{T} P C_{1}+C_{1}^{T} P C_{2} R_{4}^{-1} C_{2}^{T} P C_{1}\right) x(s) d s, \\
V_{5}(t) & =h_{2} \int_{t-h_{2}}^{t}\left(s-t+h_{2}\right) x^{T}(s)\left(R_{1}+R_{3}\right) x(s) d s, \\
V_{6}(t) & =\tau_{2} \int_{t-\tau_{2}}^{t}\left(s-t+\tau_{2}\right) x^{T}(s)\left(R_{4}+C_{2}^{T} P C_{2}\right) x(s) d s, \\
V_{7}(t) & =h_{1} \int_{t-h_{1}}^{t}\left(s-t+h_{1}\right) x^{T}(s)\left(R_{2}+R_{7}+R_{8}+R_{9}+B_{1}^{T} P B_{2} R_{3}^{-1} B_{2}^{T} P B_{1}\right) x(s) d s .
\end{aligned}
$$

Taking the operator $\mathcal{L}$ of $V_{1}(t, x(t))$ along the trajectory of system (3.1), by Lemmas 2.4 and 2.5 , we have

$$
\begin{aligned}
\mathcal{L} V_{1}(t, x(t))= & \left.2\left[x(t)+B_{1} \int_{t-h_{1}}^{t} x(s) d s\right)^{T}\right] \\
& \times P\left[\left(A+B_{1}\right) x(t)+B_{2} \int_{t-h_{2}}^{t} x(s) d s+D u(t)+f(t, x(t))\right] \\
& +\frac{1}{2} \operatorname{trace}\left[C_{1} x\left(t-\tau_{1}\right)+C_{2} \int_{t-\tau_{2}}^{t} x(s) d s\right] 2 P\left[C_{1} x\left(t-\tau_{1}\right)+C_{2} \int_{t-\tau_{2}}^{t} x(s) d s\right] \\
\leq & x^{T}(t)\left[P\left(A+B_{1}\right)+\left(A+B_{1}\right)^{T} P+P B_{2} R_{1}^{-1} B_{2}^{T} P\right. \\
& +h_{2} \int_{t-h_{2}}^{t} x^{T}(s)\left(R_{1}+R_{3}\right) x(s) d s+2 \int_{t-h_{1}}^{t} x^{T}(s) d s B_{1}^{T} P D u(t) \\
& +x^{T}\left(t-\tau_{1}\right)\left(C_{1}^{T} P C_{1}+C_{1}^{T} P C_{2} R_{4}^{-1} C_{2}^{T} P C_{1}\right) x\left(t-\tau_{1}\right) \\
& +h_{1} \int_{t-h_{1}}^{t} x^{T}(s)\left(R_{2}+B_{1}^{T} P B_{2} R_{3}^{-1} B_{2}^{T} P B_{1}\right) x(s) d s \\
& \left.\left.\left.+2 \int_{t-h_{1}}^{t} x^{T}(s) d s B_{1}^{T} P f(t, x(t))+2 x_{1}\right)\right] x(t)+2 x^{T} P D f(t) x(t)\right)+\tau_{2} \int_{t-\tau_{2}}^{t} x^{T}(s)\left(R_{4}+C_{2}^{T} P C_{2}\right) d s .
\end{aligned}
$$


By the Lemma 2.5, (2.3) and (3.5), we conclude

$$
\begin{aligned}
\mathcal{L} V_{1}(t, x(t)) \leq & x^{T}(t)\left[P\left(A+B_{1}\right)+\left(A+B_{1}\right)^{T} P+P B_{2} R_{1}^{-1} B_{2}^{T} P+P R_{5}^{-1} P\right. \\
& +\left(A+B_{1}\right)^{T} P B_{1} R_{2}^{-1} B_{1}^{T} P\left(A+B_{1}\right)+2 P D R_{10} D^{T} P \\
& \left.+P R_{6}^{-1} P+P D R_{10} D^{T} P B_{1} R_{7}^{-1} B_{1}^{T} P D R_{10} D^{T} P\right] x(t) \\
& +x^{T}\left(t-\tau_{1}\right)\left(C_{1}^{T} P C_{1}+C_{1}^{T} P C_{2} R_{4}^{-1} C_{2}^{T} P C_{1}\right) x\left(t-\tau_{1}\right) \\
+ & x^{T}\left(t-h_{1}\right)\left(R_{5}+P B_{1} R_{8}^{-1} B_{1}^{T} P\right) x\left(t-h_{1}\right) \\
+ & x^{T}\left(t-h_{2}\right)\left(R_{6}+P B_{1} R_{9}^{-1} B_{1}^{T} P\right) x\left(t-h_{2}\right) \\
+ & h_{1} \int_{t-h_{1}}^{t} x^{T}(s)\left(R_{2}+B_{1}^{T} P B_{2} R_{3}^{-1} B 2^{T} P B_{1}+R_{7}+R_{8}+R_{9}\right) x(s) d s \\
& +\tau_{2} \int_{t-\tau_{2}}^{t} x^{T}(s)\left(R_{4}+C_{2}^{T} P C_{2}\right) d s+h_{2} \int_{t-h_{2}}^{t} x^{T}(s)\left(R_{1}+R_{3}\right) x(s) d s \\
+ & 2 \alpha\left(\|P\|+h_{1}\left\|B_{1}^{T} P\right\|\right)\|x(t)\|^{2}+6\left(\|P D\|+h_{1}\left\|B_{1}^{T} P\right\|\right)\|r(t)\|_{\infty}\|x(t)\| .
\end{aligned}
$$

Taking the operator $\mathcal{L}$ of $V_{i}(t), i=2,3, \ldots, 7$ along the trajectory of system (3.1), we get

$$
\begin{aligned}
\mathcal{L} V_{2}(t)= & x^{T}(t)\left(R_{5}+P B_{1} R_{8}^{-1} B_{1}^{T} P\right) x(t) \\
& -x^{T}\left(t-h_{1}\right)\left(R_{5}+P B_{1} R_{8}^{-1} B_{1}^{T} P\right) x\left(t-h_{1}\right), \\
\mathcal{L} V_{3}(t)= & x^{T}(t)\left(R_{6}+P B_{1} R_{9}^{-1} B_{1}^{T} P\right) x(t) \\
& -x^{T}\left(t-h_{2}\right)\left(R_{6}+P B_{1} R_{9}^{-1} B_{1}^{T} P\right) x\left(t-h_{2}\right), \\
\mathcal{L} V_{4}(t)= & x^{T}(t)\left(C_{1}^{T} P C_{1}+C_{1}^{T} P C_{2} R_{4}^{-1} C_{2}^{T} P C_{1}\right) x(t) \\
& -x^{T}\left(t-\tau_{1}\right)\left(C_{1}^{T} P C_{1}+C_{1}^{T} P C_{2} R_{4}^{-1} C_{2}^{T} P C_{1}\right) x\left(t-\tau_{1}\right), \\
\mathcal{L} V_{5}(t)= & h_{2}^{2} x^{T}(t)\left(R_{1}+R_{3}\right) x(t)-h_{2} \int_{t-h_{2}}^{t} x^{T}(s)\left(R_{1}+R_{3}\right) x(s) d s, \\
\mathcal{L} V_{6}(t)= & \tau_{2}^{2} x^{T}(t)\left(R_{4}+C_{2}^{T} P C_{2}\right) x(t)-\tau_{2} \int_{t-\tau_{2}}^{t} x^{T}(s)\left(R_{4}+C_{2}^{T} P C_{2}\right) x(s) d s, \\
\mathcal{L} V_{7}(t)= & h_{1}^{2} x^{T}(t)\left(R_{2}+R_{7}+R_{8}+R_{9}+B_{1}^{T} P B_{2} R_{3}^{-1} B_{2}^{T} P B_{1}\right) x(t) \\
& -h_{1} \int_{t-h_{1}}^{t} x^{T}(s)\left(R_{2}+R_{7}+R_{8}+R_{9}+B_{1}^{T} P B_{2} R_{3}^{-1} B_{2}^{T} P B_{1}\right) x(s) d s .
\end{aligned}
$$


Combining (3.8) and (3.9), we have

$$
\begin{aligned}
&\left\llcorner V(t, x(t)) \leq x^{T}(t)[\right. P\left(A+B_{1}\right)+\left(A+B_{1}\right)^{T} P+P B_{2} R^{-1} B_{2}^{T} P+P R_{6}^{-1} P \\
&+\left(A+B_{1}\right)^{T} P B_{1} R_{2}^{-1} B_{1}^{T} P\left(A+B_{1}\right)+2 P D R_{10} D^{T} P+R_{5} \\
&+P D R_{10} D^{T} P B_{1} R_{7}^{-1} B_{1}^{T} P D R_{10} D^{T} P+h_{2}^{2}\left(R_{1}+R_{3}\right)+R_{6} \\
&+P B_{1} R_{8}^{-1} B_{1}^{T} P+P B_{1} R_{9}^{-1} B_{1}^{T} P+C_{1}^{T} P C_{2} R_{4}^{-1} C_{2}^{T} P C_{1} \\
&+h_{1}^{2}\left(R_{2}+B_{1}^{T} P B_{2} R_{3}^{-1} B_{2}^{T} P B_{1}+R_{7}+R_{8}+R_{9}\right)+C_{1}^{T} P C_{1} \\
&\left.+\tau_{2}^{2}\left(R_{4}+C_{2}^{T} P C_{2}\right)+P R_{5}^{-1} P\right] x(t)+2 \alpha h_{1}\left\|B_{1}^{T} P\right\|\|x(t)\|^{2} \\
&+ 2 \alpha\|P\|\|x(t)\|^{2}+6\left(\|P D\|+h_{1}\left\|B_{1}^{T} P\right\|\right)\|r(t)\|_{\infty}\|x(t)\| \\
& \leq-\left(\lambda_{\min }\left(Q_{1}\right)-2 \alpha\left(\|P\|+h_{1}\left\|B_{1}^{T} P\right\|\right)\right)\|x(t)\|^{2} \\
&+6\left(\|P D\|+h_{1}\left\|B_{1}^{T} P\right\|\right)\|r(t)\|_{\infty}\|x(t)\| .
\end{aligned}
$$

Let $\rho_{1}=\lambda_{\min }\left(Q_{1}\right)-2 \alpha\left(\|P\|+h_{1}\left\|B_{1}^{T} P\right\|\right), \rho_{2}=6\left(\|P D\|+h_{1}\left\|B_{1}^{T} P\right\|\right)\|r(t)\|_{\infty}$; we have

$$
\mathcal{L V}(t, x(t)) \leq-\rho_{1}\|x(t)\|^{2}+\rho_{2}\|x(t)\|
$$

Set

$$
\begin{aligned}
& \beta_{1}=\lambda_{\max }(P)+h_{1} \lambda_{\max }\left(P B_{1}\right)+h_{1} \lambda_{\max }\left(B_{1}^{T} P\right)+h_{1}^{2} \lambda_{\max }\left(B_{1}^{T} P B_{1}\right), \\
& \beta_{2}=h_{1} \lambda_{\max }\left(R_{5}+P B_{1} R_{8}^{-1} B_{1}^{T} P\right), \quad \beta_{3}=h_{2} \lambda_{\max }\left(R_{6}+P B_{1} R_{9}^{-1} B_{1}^{T} P\right), \\
& \beta_{4}=\tau_{1} \lambda_{\max }\left(C_{1}^{T} P C_{1}+C_{1}^{T} P C_{2} R_{4}^{-1} C_{2}^{T} P C_{1}\right), \quad \beta_{6}=\tau_{2}^{3} \lambda_{\max }\left(R_{4}+C_{2}^{T} P C_{2}\right), \\
& \beta_{5}=h_{2}^{3} \lambda_{\max }\left(R_{1}+R_{3}\right), \quad \beta_{7}=h_{1}^{3} \lambda_{\max }\left(R_{2}+B_{1}^{T} P B_{2} R_{3}^{-1} B_{2}^{T} P B_{1}+R_{7}+R_{8}+R_{9}\right),
\end{aligned}
$$

under an assumption that $V(t, x(t)) \leq V\left(t_{0}, x\left(t_{0}\right)\right)$ for all $t \geq t_{0}$, then

$$
\lambda_{\min }(P) \mathbb{E}\left\|x(t)+B_{1} \int_{t-h_{1}}^{t} x(s) d \mathrm{~s}\right\|^{2} \leq V(t, x(t)) \leq V\left(t_{0}, x\left(t_{0}\right)\right) \leq \sum_{i=1}^{7} \beta_{i} \mathbb{E}\|\varphi(\theta)\|^{2},
$$

so

$$
\mathbb{E}\left\|x(t)+B_{1} \int_{t-h_{1}}^{t} x(s) d s\right\|^{2} \leq \frac{\sum_{i=1}^{7} \beta_{i} \mathbb{E}\|\varphi(\theta)\|^{2}}{\lambda_{\min }(P)} .
$$


Thus, according to [21, Theorem 1.3 page 331], we have

$$
\mathbb{E}\|x(t)\|^{2} \leq\left(\frac{1+h_{1}\left\|B_{1}\right\|}{1-h_{1}\left\|B_{1}\right\|}\right)^{2} \frac{\sum_{i=1}^{7} \beta_{i} \mathbb{E}\|\varphi(\theta)\|^{2}}{\lambda_{\min }(P)} .
$$

If not, there exist $t>t_{0}$, such that $V(t, x(t)) \geq V(s, x(s))$ for all $s \in\left[t_{0}, t\right)$, and one has

$$
D^{+} \mathbb{E} V(t, x(t)) \geq 0
$$

In view of Ito's formula, we obtain

$$
D^{+} \mathbb{E} V(t, x(t))=\mathbb{E} \rho V(t, x(t)) .
$$

By (3.16) and (3.17), it is easy to derive that

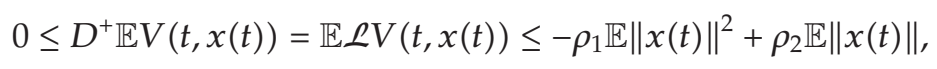

so $\mathbb{E}\|x(t)\| \leq \rho_{2} / \rho_{1}$. By (3.18), we can conclude that

$$
\mathbb{E}\|x(t)\|^{2} \leq \frac{\rho_{2}}{\rho_{1}} \mathbb{E}\|x(t)\| \leq\left(\frac{\rho_{2}}{\rho_{1}}\right)^{2}
$$

By (3.15) and (3.19), we get

$$
\mathbb{E}\|x(t)\|^{2} \leq \frac{\rho_{2}^{2}}{\rho_{1}^{2}}+\left(\frac{1+h_{1}\left\|B_{1}\right\|}{1-h_{1}\left\|B_{1}\right\|}\right)^{2} \frac{\sum_{i=1}^{7} \beta_{i} \mathbb{E}\|\varphi(\theta)\|^{2}}{\lambda_{\min }(P)},
$$

Thus

$$
\begin{aligned}
\mathbb{E}\|y(t)\|^{2} & \leq\|H\|^{2} \mathbb{E}\|x(t)\|^{2} \\
& \leq \frac{\|H\|^{2} \rho_{2}^{2}}{\rho_{1}^{2}}+\left(\frac{1+h_{1}\left\|B_{1}\right\|}{1-h_{1}\left\|B_{1}\right\|}\right)^{2} \frac{\|H\|^{2} \sum_{i=1}^{7} \beta_{i} \mathbb{E}\|\varphi(\theta)\|^{2}}{\lambda_{\min }(P)} \\
& \leq N_{1}+N_{2}\|r\|_{\infty}^{2}
\end{aligned}
$$

where

$$
N_{1}=\left(\frac{1+h_{1}\left\|B_{1}\right\|}{1-h_{1}\left\|B_{1}\right\|}\right)^{2} \frac{\sum_{i=1}^{7} \beta_{i}\|H\|^{2}}{\lambda_{\min }(P)} \mathbb{E}\|\varphi(\theta)\|^{2}, \quad N_{2}=\frac{36\|H\|^{2}\left(\|P D\|+h_{1}\left\|B_{1}^{T} P\right\|\right)}{\lambda_{\min }\left(Q_{1}\right)-2 \alpha\left(\|P\|-h_{1}\left\|B_{1}^{T} P\right\|\right)} .
$$

By Definition 2.2, the nonlinear stochastic control system (3.1) with the control law (2.3) is said to be BIBO stabilized in mean square. This completes the proof. 
If we transform the original system (2.1) to the following system

$$
\begin{aligned}
d\left[x(t)+B_{2} \int_{t-h_{2}}^{t}\left(s-t+h_{2}\right) x(s) d s\right]= & {\left[\left(A+h_{2} B_{2}\right) x(t)+B_{1} x\left(t-h_{1}\right)+f(t, x(t))\right] d t+D u(t) d t } \\
& +\left[C_{1} x\left(t-\tau_{1}\right)+C_{2} \int_{t-\tau_{2}}^{t} x(s) d s\right] d \mathbf{w}(t), \quad t \geq t_{0} \geq 0 \\
y(t)= & H x(t) \\
x(\theta)= & \varphi(\theta) \in C_{\mathcal{\mp}_{0}}^{b}\left(\left[t_{0}-\tau, t_{0}\right] ; R^{n}\right), \quad \theta \in\left[t_{0}-\tau, t_{0}\right],
\end{aligned}
$$

we can get the following result.

Theorem 3.2. The nonlinear stochastic control system (3.23) with the control law (2.3) is BIBO stabilized in mean square if there exist symmetric positive-definite matrices $S_{i}>0, i=1,2, \ldots, 10$, and $Q_{2}>0$ such that

$$
\lambda_{\min }\left(Q_{2}\right)-2 \alpha\|\widehat{P}\|>0
$$

and $\widehat{P}$ is the symmetric positive solution of the Riccati equation

$$
\widehat{P}\left(A+h_{2} B_{2}\right)+\left(A+h_{2} B_{2}\right)^{T} \widehat{P}+\widehat{P} \Sigma_{2} \widehat{P}+\Delta_{2}+\Xi_{2}=-Q_{2}
$$

where

$$
\begin{aligned}
\Sigma_{2}= & B_{1} S_{2}^{-1} B_{1}^{T}+2 D S_{10} D^{T}+S_{5}^{-1}+S_{6}^{-1} \\
\Delta_{2}= & \left(A+h_{2} B_{2}\right)^{T} \widehat{P} B_{2} S_{1}^{-1} B_{2}^{T} \widehat{P}\left(A+h_{2} B_{2}\right)+D^{T} \widehat{P} S_{7}^{-1} \widehat{P} D+\tau_{2}^{2} C_{2}^{T} \widehat{P} C_{2} \\
& +D^{T} \widehat{P} S_{8}^{-1} \widehat{P} D+C_{1}^{T} \widehat{P} C_{1}+C_{1}^{T} \widehat{P} C_{2} S_{4}^{-1} C_{2}^{T} \widehat{P} C_{1}+D^{T} \widehat{P} S_{9}^{-1} \widehat{P} D \\
& +B_{1}^{T} \widehat{P} B_{2} S_{3}^{-1} B_{2}^{T} \widehat{P} B_{1} \\
\Xi_{2}= & S_{2}+S_{5}+S_{6}+\frac{1}{3} h_{2}^{4}\left(S_{1}+S_{3}+S_{7}+S_{8}+S_{9}\right)+\tau_{2}^{2} S_{4}, \\
K_{1}= & S_{10} D^{T} \widehat{P}, \quad K_{2}=K_{3}=D^{-1} .
\end{aligned}
$$

Proof. We define a Lyapunov functional $V(t, x(t))$ as

$$
V(t, x(t))=V_{1}(t, x(t))+V_{2}(t)+V_{3}(t)+V_{4}(t)+V_{5}(t)+V_{6}(t)
$$


where

$$
\begin{aligned}
V_{1}(t, x(t)) & =\left(x(t)+B_{2} \int_{t-h_{2}}^{t}\left(s-t+h_{2}\right) x(s) d s\right)^{T} \widehat{P}\left(x(t)+B_{2} \int_{t-h_{2}}^{t}\left(s-t+h_{2}\right) x(s) d s\right), \\
V_{2}(t) & =\int_{t-h_{1}}^{t} x^{T}(s)\left(S_{2}+S_{5}+B_{1}^{T} \widehat{P} B_{2} S_{3}^{-1} B_{2}^{T} \widehat{P} B_{1}+D^{T} \widehat{P} S_{8}^{-1} \widehat{P} D\right) x(s) d s, \\
V_{3}(t) & =\int_{t-h_{2}}^{t} x^{T}(s)\left(S_{6}+D^{T} \widehat{P} S_{9}^{-1} \widehat{P} D\right) x(s) d s, \\
V_{4}(t) & =\int_{t-\tau_{1}}^{t} x^{T}(s)\left(C_{1}^{T} \widehat{P} C_{1}+C_{1}^{T} \widehat{P} C_{2} S_{4}^{-1} C_{2}^{T} \widehat{P} C_{1}\right) x(s) d s, \\
V_{5}(t) & =\tau_{2} \int_{t-\tau_{2}}^{t}\left(s-t+\tau_{2}\right) x^{T}(s)\left(S_{4}+C_{2}^{T} \widehat{P} C_{2}\right) x(s) d s, \\
V_{6}(t) & =\frac{1}{3} \int_{t-h_{2}}^{t}\left(s-t+h_{2}\right)^{3} x^{T}(s)\left(S_{1}+S_{3}+S_{7}+S_{8}+S_{9}\right) x(s) d s .
\end{aligned}
$$

Let $\left.\rho_{1}=\lambda_{\min }\left(Q_{2}\right)-2 \alpha\left(1+h_{2}^{2}\right)\|\widehat{P}\|\right), \rho_{2}=6\left(1+h_{2}^{2}\right)\|\widehat{P} D\|\|r(t)\|_{\infty}$. The rest of the proof is essentially as that of Theorem 3.1, and hence is omitted. This completes the proof.

Remark 3.3. If we transform the original system (2.1) to the following system

$$
\begin{aligned}
d[x(t) & \left.+B_{1} \int_{t-h_{1}}^{t} x(s) d s+B_{2} \int_{t-h_{2}}^{t}\left(s-t+h_{2}\right) x(s) d s\right] \\
= & {[A x(t)+f(t, x(t))+D u(t)] d t } \\
& +\left[C_{1} x\left(t-\tau_{1}\right)+C_{2} \int_{t-\tau_{2}}^{t} x(s) d s\right] d \mathbf{w}(t), \quad t \geq t_{0} \geq 0 \\
y(t)= & H x(\mathrm{t}) \\
x(\theta)= & \varphi(\theta) \in C_{q_{0}}^{b}\left(\left[t_{0}-\tau, t_{0}\right] ; R^{n}\right), \quad \theta \in\left[t_{0}-\tau, t_{0}\right],
\end{aligned}
$$

using the same process of Theorem 3.1, we can get the corresponding BIBO stability in mean square results. Here we omitted it.

Theorem 3.4. The nonlinear stochastic control system (2.1) with the control law (2.3) is BIBO stabilized in mean square if there exist symmetric positive-definite matrices $\Omega_{i}>0, i=1,2, \ldots, 6$, and $Q_{3}>0$ such that

$$
\lambda_{\min }\left(Q_{3}\right)-2 \alpha\|\tilde{P}\|>0
$$


and $\widetilde{P}$ is the symmetric positive solution of the Riccati equation

$$
\widetilde{P} A+A^{T} \widetilde{P}+\widetilde{P} \Sigma_{3} \widetilde{P}+\Delta_{3}+\Xi_{3}=-Q_{3}
$$

where

$$
\begin{aligned}
& \Sigma_{3}=B_{1} \Omega_{1}^{-1} B_{1}^{T}+h_{2} B_{2} \Omega_{2}^{-1} B_{2}^{T}+2 D \Omega_{6} D^{T}+\Omega_{4}^{-1}+\Omega_{5}^{-1}, \\
& \Delta_{3}=C_{1}^{T} \tilde{P} C_{1}+\tau_{2} C_{2}^{T} \tilde{P} C_{1} \Omega_{3}^{-1} C_{1}^{T} \tilde{P} C_{2}+\tau_{2}^{2} C_{2}^{T} \tilde{P} C_{2}, \\
& \Xi_{3}=\Omega_{1}+h_{2} \Omega_{2}+\tau_{2} \Omega_{3}+\Omega_{4}+\Omega_{5}, \\
& K_{1}=\Omega_{6} D^{T} \tilde{P}, \quad K_{2}=K_{3}=D^{-1} .
\end{aligned}
$$

Proof. We define a Lyapunov functional $V(t, x(t))$ as

$$
V(t, x(t))=V_{1}(t, x(t))+V_{2}(t)+V_{3}(t)+V_{4}(t)+V_{5}(t),
$$

where

$$
\begin{aligned}
V_{1}(t, x(t)) & =x(t)^{T} \tilde{P} x(t), \\
V_{2}(t) & =\int_{t-h_{1}}^{t} x^{T}(s)\left(\Omega_{1}+\Omega_{4}\right) x(s) d s+\int_{t-h_{2}}^{t} x^{T}(s) \Omega_{5} x(s) d s, \\
V_{3}(t) & =\int_{t-h_{2}}^{t}\left(s-t+h_{2}\right) x^{T}(s) \Omega_{2} x(s) d s \\
V_{4}(t) & =\int_{t-\tau_{1}}^{t} x^{T}(s)\left(C_{1}^{T} \tilde{P} C_{1}+\tau_{2} C_{2}^{T} \tilde{P} C_{1} \Omega_{3}^{-1} C_{1}^{T} \tilde{P} C_{2}\right) x(s) d s \\
V_{5}(t) & =\int_{t-\tau_{2}}^{t}\left(s-t+\tau_{2}\right) x^{T}(s)\left(\Omega_{3}+\tau_{2} C_{2}^{T} \tilde{P} C_{2}\right) x(s) d s
\end{aligned}
$$

Let $\left.\rho_{1}=\lambda_{\min }\left(Q_{3}\right)-2 \alpha\|\tilde{P}\|\right), \rho_{2}=6\|\tilde{P} D\|\|r(t)\|_{\infty}$. The following proof runs as that of Theorem 3.1., and hence is omitted. This completes the proof.

Remark 3.5. The systems (2.1), (3.1), and (3.23) are also asymptotically stable in mean square when all the conditions in Theorems 3.1-3.4 are satisfied, if $r(t)=0, r\left(t-h_{1}\right)=0, r\left(t-h_{2}\right)=0$ in (2.3).

Remark 3.6. In [18], the authors studied the BIBO stabilization of stochastic delayed systems with uncertainty in terms of Razumikhin technique and comparison principle. In the present paper, we are the first to introduce a new way in the study of BIBO stabilization for stochastic delayed systems by using algebraic Riccati matrix equation, which makes the stability conditions be morefeasible. 
Remark 3.7. In [18], researchers investigate the BIBO stabilization of stochastic delayed systems with uncertainty without distributed time delays and nonlinear perturbations. In [15], the authors discussed the BIBO stabilization of mixed time-delayed systems with nonlinear perturbations, but the environmental noise is not taken into account in the models. Therefore, compared with (see [14-18]), the systems reported in this paper are more general.

Remark 3.8. The criteria given in Theorem 3.1-3.4 are delay dependent with respect to delays. Generally speaking, the delay-dependent stability criterion is less conservative than delayindependent stability when the time delay is small.

\section{Example}

In this section, a numerical example will be presented to show the effectiveness of the main results derived in this paper.

Example 4.1. As a simple application of Theorem 3.4, consider the stochastic control system (2.1) with control law (2.3); the parameters are given by

$$
\begin{aligned}
& A=\left(\begin{array}{cc}
-2 & 0 \\
0 & -1
\end{array}\right), \quad B_{1}=\left(\begin{array}{cc}
-1 & 1 \\
1 & -2
\end{array}\right), \quad B_{2}=C_{1}=\left(\begin{array}{ll}
1 & 0 \\
0 & 1
\end{array}\right), \quad D=\left(\begin{array}{cc}
-1 & 0 \\
0 & -1
\end{array}\right), \\
& C_{2}=0, f=[0.25 \sin (t), 0.25 \cos (t)]^{T} .
\end{aligned}
$$

For

$$
\Omega_{i}=\left(\begin{array}{ll}
1 & 0 \\
0 & 1
\end{array}\right) \quad i=1,2, \ldots, 6, \quad Q_{3}=\left(\begin{array}{cc}
11 & 7 \\
7 & 14
\end{array}\right)
$$

solving for $\widetilde{P}$ in the Riccati matrix (3.31) gives us

$$
P=\left(\begin{array}{ll}
1 & 1 \\
1 & 4
\end{array}\right)
$$

therefore, the stabilizing feedback gain matrix is given by

$$
K_{1}=\left(\begin{array}{cc}
-11 & -7 \\
-7 & -14
\end{array}\right), \quad K_{2}=K_{3}=\left(\begin{array}{cc}
-1 & 0 \\
0 & -1
\end{array}\right) \text {. }
$$

Meanwhile, we obtain the maximum value $\tau_{\max }=0.6667$.

\section{Conclusions}

The problem of delay-dependent BIBO stabilization in mean square for the stochastic control systems with mixed delays and nonlinear perturbations was investigated. A suitable class of 
Lyapunov functional combined with the descriptor model transformation and decomposition technique of controller were constructed to derive some novel mean square BIBO stability criteria. This paper was the first to successfully introduce the method of Riccati matrix equation to stochastic BIBO stabilization. A numerical example was given to illustrate the validity of the main results.

\section{Acknowledgments}

The authors would like to thank the editor and the reviewers for their detailed comments and valuable suggestions which have led to a much improved paper. This work was supported by the National Natural Science Foundation of China (Grant no. 60736029) and the National Basic Research Program of China (2010CB732501).

\section{References}

[1] S. Kotsios and O. Feely, "A BIBO stability theorem for a two-dimensional feedback discrete system with discontinuities," Journal of the Franklin Institute B, vol. 335, no. 3, pp. 533-537, 1998.

[2] T. Bose and M. Q. Chen, "BIBO stability of the discrete bilinear system," Digital Signal Processing, vol. 5, no. 3, pp. 160-166, 1995.

[3] J. R. Partington and C. Bonnet, " $L_{\infty}$ and BIBO stabilization of delay systems of neutral type," Systems and Control Letters, vol. 52, no. 3-4, pp. 283-288, 2004.

[4] K. H. You and E. B. Lee, "BIBO stability integral ( $L_{\infty}$-gain) for second-order systems with numerator dynamics," Automatica, vol. 36, no. 11, pp. 1693-1699, 2000.

[5] A. T. Tomerlin and W. W. Edmonson, "BIBO stability of D-dimensional filters," Multidimensional Systems and Signal Processing, vol. 13, no. 3, pp. 333-340, 2002.

[6] W. Wang and Y. Zou, "The stabilizability and connections between internal and BIBO stability of 2-D singular systems," Multidimensional Systems and Signal Processing, vol. 15, no. 1, pp. 37-50, 2004.

[7] Z.-P. Ma, H.-F. Huo, and C.-Y. Liu, "Stability and Hopf bifurcation analysis on a predator-prey model with discrete and distributed delays," Nonlinear Analysis: Real World Applications, vol. 10, no. 2, pp. 1160-1172, 2009.

[8] G. Lin and R. Yuan, "Periodic solution for a predator-prey system with distributed delay," Mathematical and Computer Modelling, vol. 42, no. 9-10, pp. 959-966, 2005.

[9] H. Guo and L. Chen, "The effects of impulsive harvest on a predator-prey system with distributed time delay," Communications in Nonlinear Science and Numerical Simulation, vol. 14, no. 5, pp. 2301-2309, 2009.

[10] D. Xu, S. Zhong, and M. Li, “BIBO stabilization of large-scale systems," Control Theory E Applications, vol. 12, no. 6, pp. 758-763, 1995.

[11] D. Y. Xu and S. M. Zhong, "BIBO stabilization of multivariable feedback systems," Journal of University of Electronic Science and Technology of China, vol. 24, no. 1, pp. 90-96, 1995.

[12] S. M. Zhong and Y. Q. Huang, "BIBO stabilization of nonlinear system with time-delay," Journal of University of Electronic Science and Technology of China, vol. 32, no. 6, pp. 655-657, 2000.

[13] K. C. Cao, S. M. Zhong, and B. S. Liu, "BIBO and robust stabilization for system with time-delay and nonlinear perturbations," Journal of University of Electronic Science and Technology of China, vol. 32, no. 6, pp. 787-789, 2003.

[14] P. Li and S.-M. Zhong, "BIBO stabilization of time-delayed system with nonlinear perturbation," Applied Mathematics and Computation, vol. 195, no. 1, pp. 264-269, 2008.

[15] P. Li and S.-M. Zhong, "BIBO stabilization for system with multiple mixed delays and nonlinear perturbations," Applied Mathematics and Computation, vol. 196, no. 1, pp. 207-213, 2008.

[16] P. Li and S.-M. Zhong, "BIBO stabilization of piecewise switched linear systems with delays and nonlinear perturbations," Applied Mathematics and Computation, vol. 213, no. 2, pp. 405-410, 2009.

[17] P. Li, S.-M. Zhong, and J.-Z. Cui, “Delay-dependent robust BIBO stabilization of uncertain system via LMI approach," Chaos, Solitons E Fractals, vol. 40, no. 2, pp. 1021-1028, 2009. 
[18] Y. Fu and X. Liao, "BIBO stabilization of stochastic delay systems with uncertainty," IEEE Transactions on Automatic Control, vol. 48, no. 1, pp. 133-138, 2003.

[19] K. Gu, "An integral inequality in the stability problem of time-delay systems," Proceedings of the IEEE Conference on Decision and Control, vol. 3, pp. 2805-2810, 2000.

[20] Z. Wang, H. Shu, Y. Liu, D. W.C. Ho, and X. Liu, "Robust stability analysis of generalized neural networks with discrete and distributed time delays," Chaos, Solitons E Fractals, vol. 30, no. 4, pp. 886896, 2006.

[21] V. Kolmanovskii and A. Myshkis, Introduction to the Theory and Applications of Functional-Differential Equations, vol. 463 of Mathematics and Its Applications, Kluwer Academic, Dordrecht, The Netherlands, 1999. 